\title{
Influence of sweeping detonation-wave loading on shock hardening and damage evolution during spallation loading in tantalum
}

\author{
G.T. Gray III, L.M. Hull, V. Livescu, J.R. Faulkner, M.E. Briggs, and E.K. Cerreta \\ Los Alamos National Laboratory, Los Alamos, New Mexico 87544, USA
}

\begin{abstract}
Widespread research over the past five decades has provided a wealth of experimental data and insight concerning the shock hardening, damage evolution, and the spallation response of materials subjected to square-topped shock-wave loading profiles. However, fewer quantitative studies have been conducted on the effect of direct, in-contact, high explosive (HE)driven Taylor wave (unsupported shocks) loading on the shock hardening, damage evolution, or spallation response of materials. Systematic studies quantifying the effect of sweeping-detonation wave loading are yet sparser. In this study, the shock hardening and spallation response of Ta is shown to be critically dependent on the peak shock stress and the shock obliquity during sweeping-detonation-wave shock loading. Sweeping-wave loading is observed to: a) yield a lower spall strength than previously documented for 1-D supported-shock-wave loading, b) exhibit increased shock hardening as a function of increasing obliquity, and c) lead to an increased incidence of deformation twin formation with increasing shock obliquity.
\end{abstract}

\section{Introduction}

Over the past five decades numerous studies have provided a wealth of experimental data and insight concerning shock hardening and the spallation response of materials subjected to square-topped shock-wave loading profiles [1-3]. Fewer researchers have quantified the effect of direct, in-contact, high explosive (HE)-driven Taylor wave (or triangular-wave) loading on the shock hardening, damage evolution, or spallation response of materials $[4,5]$. Direct in-contact high-explosive loading induces an impulse dubbed a "Taylor Wave". Direct explosive loading imparts a significantly different loading history than that achieved by a square-topped impulse in terms of both the pulse duration at a fixed peak pressure, and a different unloading strain rate from the peak Hugoniot state achieved [6]. Seminal studies by Rinehart [7], and Butcher et al. [6] detailed how the formation and thickness of the "scabbing under explosive loading" (i.e. spallation), depends directly on the shape of the stress wave imposed. Butcher et al. [6] demonstrated that "since spallation is studied by introducing a pulse and observing the effects of the tensile stresses within the material, knowledge of the stress history at the spall plane is necessary to the quantitative understanding of spall". Further, experiments by Butcher et al. [6] revealed "that the spallation stress is less for square pulses than for triangular pulses".

Later studies by Al'tshuler, Novikov, and Divnov [8] summarized direct explosively-driven spallation experiments stating that "the resistance of metal to fracture in the case of a strong blow or explosion is not a constant characteristic of its strength, but rather may vary over a wide range depending on the pressure gradient (or on strain rate) in the interacting rarefaction waves". Additional experiments by Drummond [9] and more recently Ogorodnikov et al. [10] has led to an increased emphasis on research in HE-driven spallation due to oblique loading via sliding detonation waves.

Recent sweeping-detonation-wave shock-loading and spallation experiments on $\mathrm{Cu}$ have shown that based on variations in the specifics of the shock drive (pulse shape, peak stress, shock obliquity) and sample geometry, "spall strength" in High-purity $\mathrm{Cu}$ varies by over a factor of two and the details of the mechanisms of the damage evolution are seen to vary [2]. Divergent direct PETN explosive loading of Ta revealed that Ta twin formation during shock loading in Ta is a strong function of shock obliquity. This is consistent with the effect of obliquity on the imposed stress tensor during shock loading that effectively varies the ratio of the spherical (hydrostatic) and deviatoric (shear) stress $[3,11]$.

\section{Experimental procedure}

Targets for sweeping detonation-wave loading tests were prepared from commercially pure, triple electron-beam melted and annealed tantalum plate, $10.2 \mathrm{~mm}$ in thickness obtained from Cabot Corporation. The chemical composition (in wt $\%$ ) was analyzed to be carbon $10 \mathrm{ppm}$, oxygen $<50 \mathrm{ppm}$, nitrogen $<10 \mathrm{ppm}$, hydrogen $<5 \mathrm{ppm}$, tungsten $<25 \mathrm{ppm}$, niobium $<25 \mathrm{ppm}$, titanium $<5 \mathrm{ppm}$, iron $<5 \mathrm{ppm}$, and the balance Ta. The plate was produced from an ingot, which was forged into a billet; this was then annealed, and subsequently cut prior to cross rolling. The plates were straight rolled in the final finishing passes. Cross rolling of the Ta plate resulted in divergence from the typical straight-rolled texture, from a partial to a nearly continuous $\gamma$ fiber $(<111>/ / N D)$ along with the virtual extinction of the partial $\alpha$ fiber $(<110>/ / R D)$. The texture strength was moderate, with the $<111>$ fiber component approximately eight times random. Acoustic properties of the tantalum were measured using $5 \mathrm{MHz}$ quartz transducers with a Parametrics 500PR pulse receiver. The density $\left(\rho_{0}\right)$ of the tantalum studied was measured to be $16.58 \pm$ $0.01 \mathrm{~g} / \mathrm{cc}$, longitudinal sound speed $\left(c_{L}\right) 4.13 \pm 0.03 \mathrm{~mm} / \mu \mathrm{s}$ and shear sound speed $\left(c_{S}\right) 2.04 \pm 0.03 \mathrm{~mm} / \mu \mathrm{s}$. The Poisson ratio $(v)$ was 0.339 . The explosive assembly used to drive the sweeping wave into the tantalum samples is shown in figure 1. The samples were $10.2 \mathrm{~mm}$ thick 


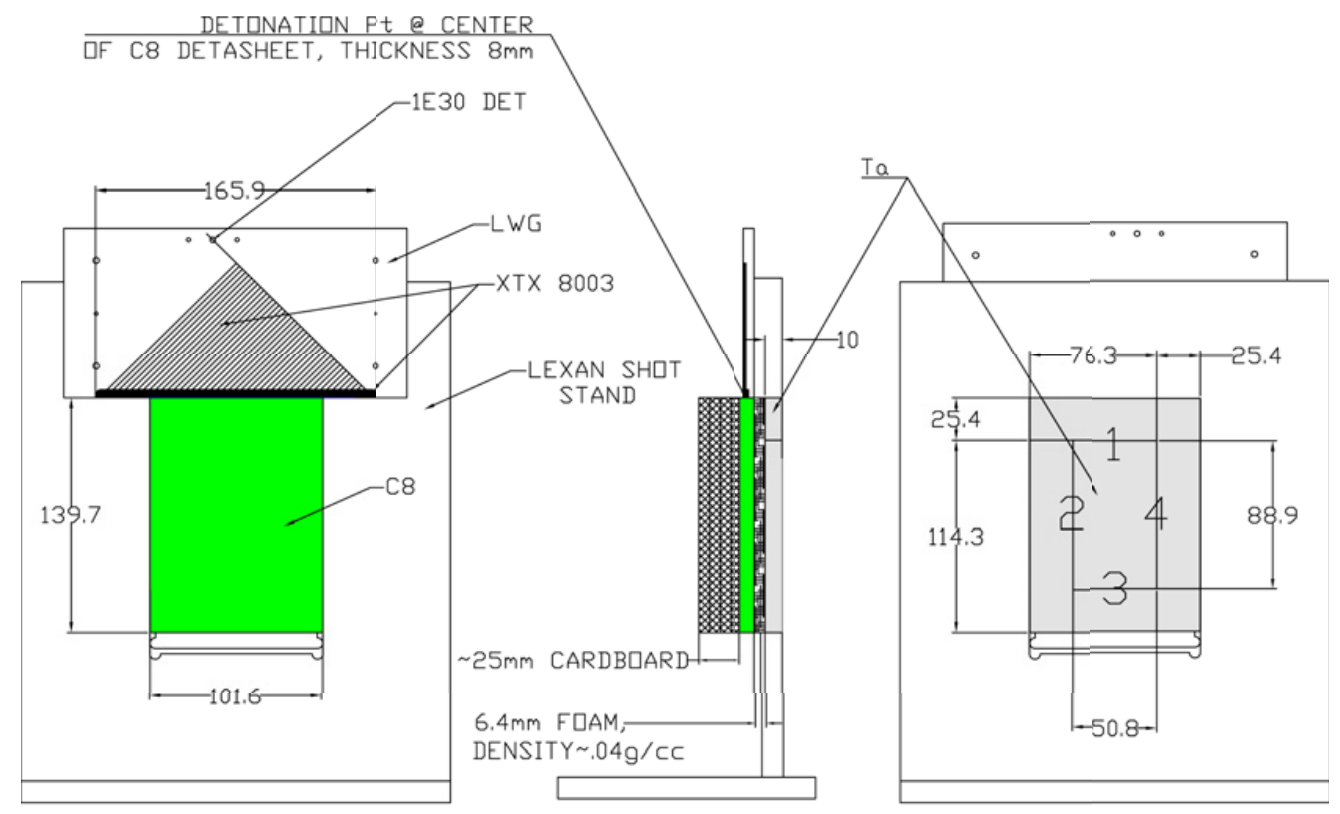

Fig. 1. Three schematic views of sweeping detonation-wave shot set-up.

$\times 50.8 \mathrm{~mm}$ wide $\times 88.9 \mathrm{~mm}$ long. Equal thickness rails made of tantalum that are $25.4 \mathrm{~mm}$ in width by $10.2 \mathrm{~mm}$ in thickness surrounded the sample to provide relief from the transverse and end (longitudinal) rarefactions along the edges that tend to cause the edges to lag.

The explosive drive was provided by Detasheet initiated with a line wave generator. The line wave generator is made by extruding XTX explosive into tracks that form equal lateral triangles. The line wave generators have a small arrival time ripple on the output wave that corresponds to the spacing between the tracks, and the average output simultaneity is $\pm 10 \mathrm{~ns}$. Three explosive loading configurations were used to approach conditions of incipient spall. All of the experiments used $8 \mathrm{~mm}$ of Detasheet explosive, with varying amounts of polyurethane foam between the explosive and the tantalum. The plate thickness and the lateral dimensions of the assembly were not varied. In the first configuration an $8 \mathrm{~mm}$ thick piece of Detasheet was separated from the tantalum by a $6.35 \mathrm{~mm}$ layer of $0.04 \mathrm{~g} / \mathrm{cc}$ polyurethane foam. The second configuration used $8 \mathrm{~mm}$ of Detasheet that was separated from the tantalum by a $3.175 \mathrm{~mm}$ layer of $0.25 \mathrm{~g} / \mathrm{cc}$ polyurethane foam. In the third shot, the $8 \mathrm{~mm}$ of Detasheet was placed in direct contact with the tantalum assembly. The purpose of the foam is to allow the explosive products to expand before interacting with the sample, thereby producing a reduced amplitude oblique shock in the sample.

The primary diagnostics for these experiments was sample recovery with post-mortem metallurgical analysis and Photon Doppler Velocimetry (PDV). The PDV was used to capture the velocity history of the sample at four points in an approximately square array. The probes have a $100 \mathrm{~mm}$ working distance and each probe interrogates a spot that is about $200 \mu \mathrm{m}$ in diameter. The array is roughly aligned so that two pairs of points are approximately parallel to the detonation direction and the two pairs straddle the center of the sample. The probe locations were placed at 50 and $100 \mathrm{~mm}$ from the top surface of the Ta sample.
The alignment is not expected to be exact with regard to the probe's interrogation points, but adequately perpendicular to the tantalum surface to capture good return signals. The objective of the recovery was to identify and characterize the micro-mechanisms of damage evolution and observe the evolution in damage evolution during spall driven by a sweeping wave; i.e., as a function of increasing shock obliquity. The objective of the velocimetry was to characterize the free surface velocity wave profiles and their differences as a function of variations in explosive drive.

The Ta specimens following sweeping-wave loading were cross-sectioned and prepared for optical metallography and electron backscatter diffraction (EBSD) [12]. Automated EBSD scans were performed with a step size of $0.15 \mu \mathrm{m}$ in a hexagonal grid at $20 \mathrm{kV}$ in an FEI XL30 FEG-SEM equipped with TSL data acquisition hardware and software. The local misorientations as a function of location surrounding areas of interest were mapped.

\section{Results and discussion}

\subsection{Post-mortem metallurgical analysis}

The damage evolution in energetic-driven oblique shock loading of Ta was seen to vary as a function of the imposed explosive loading intensity. Figure 2 shows macroscopic optical micrographs of the three sweeping-wave loaded samples as a function of the explosive loading set-up. Optical microscopy on tantalum specimen $\boldsymbol{I}$ indicated no defined spall plane and very incipient void damage evolved in the sample. Isolated voids up to $10 \mu \mathrm{m}$ in diameter were scattered throughout the microstructure as seen in figure 3 .

The majority of these voids appear to lie on or near grain boundaries. In response to the diverging detonation wave, Ta specimen $\boldsymbol{I}$ displayed a higher density of twins near the bottom surface, away from the surface adjacent 

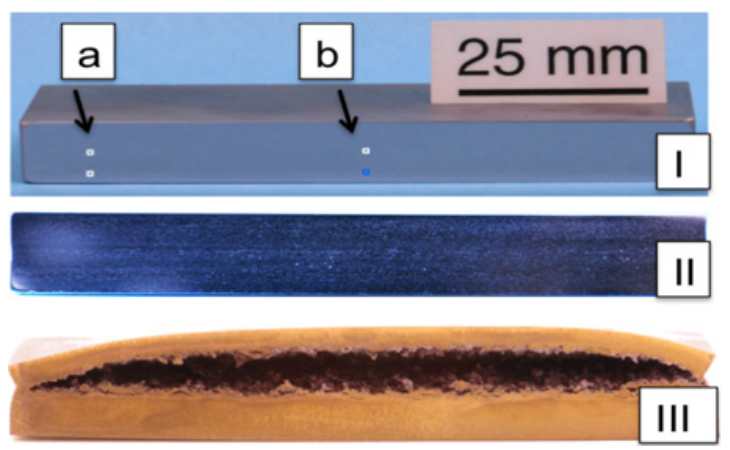

Fig. 2. Macroscopic cross-sectional view of the three sweeping detonation-wave samples: I) $8 \mathrm{~mm}$ of Detasheet with a $6.35 \mathrm{~mm}$ foam interlayer revealing no observable evolved damage, II) $8 \mathrm{~mm}$ of Detasheet with $3.175 \mathrm{~mm}$ of foam interlayer showing developing incipient spall including partial linkage and cracking between voids, and III) significant incipient spall including a single spall scab and extensive ductile tearing. The direction of the sweeping wave is from left to right in each sample.

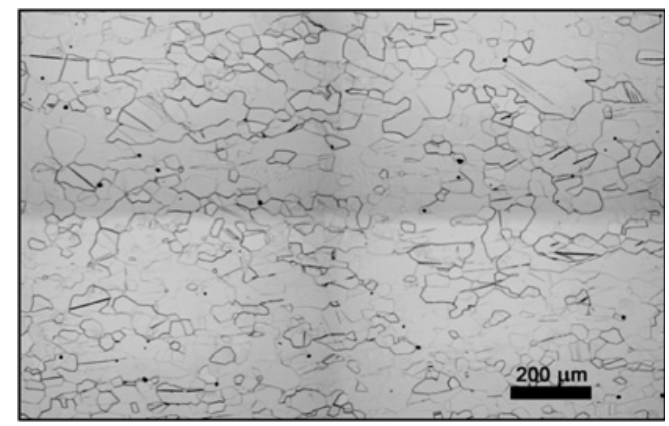

Fig. 3. Isolated voids and dislocation pits in specimen $\boldsymbol{I}$.

to the explosive. This is where the loading obliquity was maximum. Electron Backscattered Diffraction was used to interrogate specific regions for detection of obliquity effects as a function of distance from the detonation point on Ta specimen $\boldsymbol{I}$. Optical metallography of specimen $\boldsymbol{I}$ following etching and viewed using polarized illumination also revealed extensive dislocation pits as seen in figure 4 . Preferential chemical attack due to small regions of stress with a subtly different electrode potential resolves these pits [13]. The propensity of dislocation pits to lie at or near grain boundaries is consistent with the need to plastically accommodate the compatibility stresses between polycrystals.

For EBSD analysis, we selected two regions, approximately $50 \mathrm{~mm}$ apart along the $\mathrm{x}$ direction. The two locations are denoted as positions ' $a$ ' and ' $b$ ' in figure 2. Two sets of scans with a step size of 0.7 microns were performed. The EBSD data indicated no microstructural differences between regions ' $a$ ' and ' $b$ ' as seen in figure 5. EBSD data analysis for specimen $\boldsymbol{I}$ also revealed essentially no microstructural disturbances around voids and minimal deformation affected material aside from the presence of twins. The usual defect indicators sought were: 1) grain orientation gradients as witnesses of anisotropic deformation, 2) enhanced localized misorientations as potential damage initiation sites, and 3) deformation twins as suggesting a change in the balance of hydrostatic and

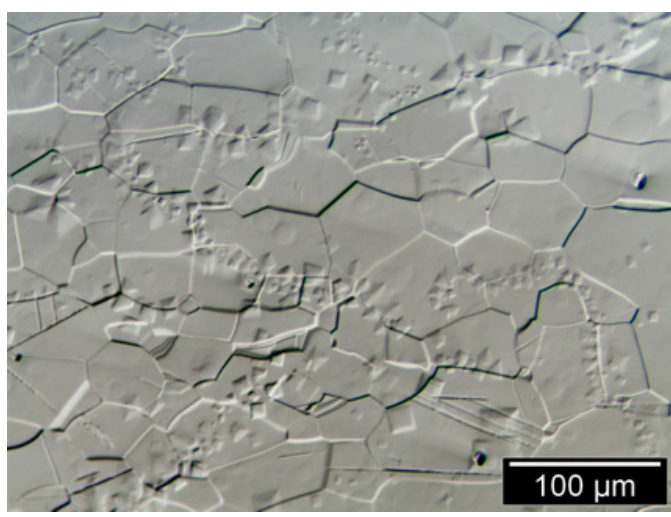

Fig. 4. Optical metallography of Ta Specimen $\boldsymbol{I}$ using polarized illumination revealing dislocation etch pits preferentially located along grain boundaries.

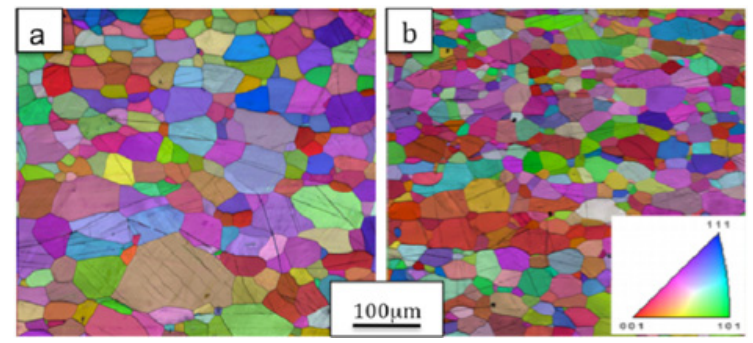

Fig. 5. EBSD scans of regions 'a' and 'b' from Ta Specimen $\boldsymbol{I}$ showing minimal microstructural damage evolution due to the sweeping-wave loading with $8 \mathrm{~mm}$ Detasheet plus $6.35 \mathrm{~mm}$ foam.

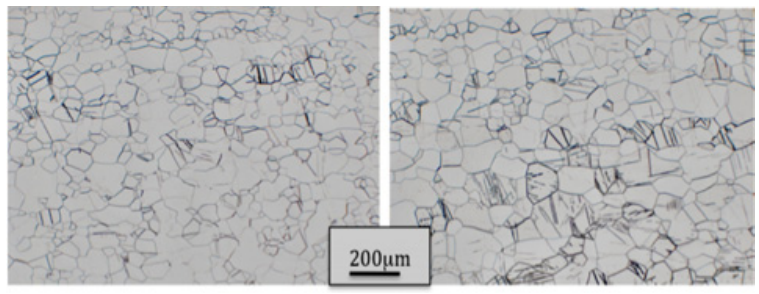

Fig. 6. Increasing propensity of deformation twin formation in Ta specimen $\boldsymbol{I I}$ as a function of increasing shock obliquity (shock obliquity increasing from left to right).

deviatoric stresses as well as potential sites for damage nucleation. However, both the crystal orientation maps and the kernel average misorientation maps were found to be similar for the two regions interrogated in specimen $\boldsymbol{I}$.

As minimal incipient spallation damage was found in specimen $\boldsymbol{I}$, the amount of the foam interlayer was reduced in the second experiment to $3.175 \mathrm{~mm}$ while keeping the explosive thickness unchanged. Specimen $\boldsymbol{I I}$ was found to exhibit a more advanced damage state and a vaguely defined spall plane as seen in figure 2. Voids were up to $200 \mu \mathrm{m}$ in diameter. While larger voids in specimen II appear round, incipient damage areas indicate that they are the result of growth and coalescence of multiple small voids. In response to the diverging detonation wave, both specimens $\boldsymbol{I}$ and $\boldsymbol{I I}$ displayed a higher density of twins near the bottom edge compared to the top, and closer to the right bottom corner away from the detonation edge similar to our previous studies on $\mathrm{Cu}$ and $\mathrm{Ta}$ $[2,3]$. Figure 6 shows the increased density of deformation 

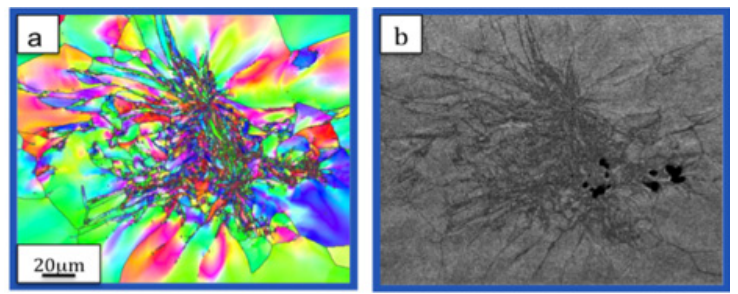

Fig. 7. Incipient damage région in spécimen II showing damaged région in a) crystal orientation and b) SEM detector signal maps. The orientation triangle is the same as in figure 5 .

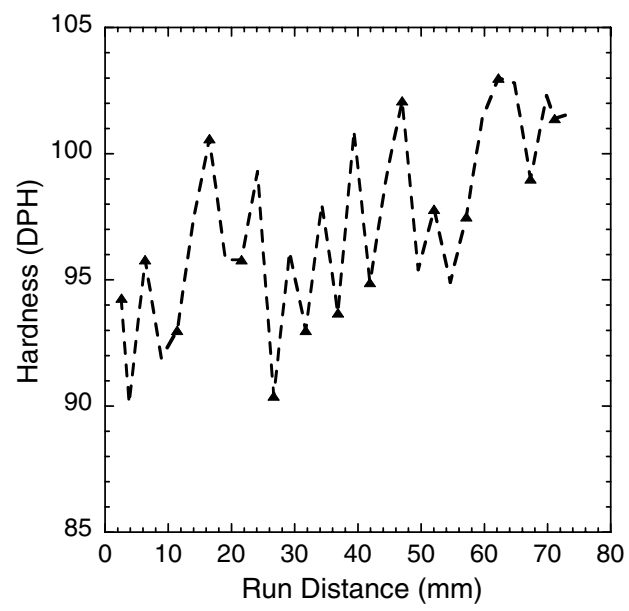

Fig. 8. Hardness measurements of specimen $\boldsymbol{I I}$ as a function of detonation run distance along the sample reflecting the effect of shock obliquity on shock hardening.

twins formed in specimen $\boldsymbol{I I}$ as a function of increased shock obliquity coincidently while the peak 'pressure' or hydrostatic component of the experiment is falling with increasing shock obliquity [11].

Incipient regions of strain localization as shown in figure 7 for specimen II indicate large areas of damageaffected material. Using the kernel-average misorientation (KAM map), one can identify multiple "seeds" for such localization. Due to their proximity, these seeds begin to interact with each other creating large deformationaffected zones offering multiple void nucleation sites. Such highly disturbed regions, with intragranular orientation gradients of up to $45^{\circ}$, lead to multiple voids nucleating and growing simultaneously.

The influence of shock obliquity on shock hardening in specimen II was probed by measuring the diamondpyramid hardness of the Ta sample $1 \mathrm{~mm}$ below the loading surface as a function of run distance along the sample length. In figure 8 the hardness is seen to increase as a function of run distance (shock wave obliquity). Increasing hardness as a function of run distance subjected to sweeping-detonation-wave loading is thought to reflect a higher dislocation density consistent with increasing shear stresses with obliquity. Increasing shear stresses as a function of obliquity while the "hydrostatic pressure" portion of the imposed stress tensor decreases is the same trend as previously shown in Ta twinning studies of sweeping-wave loading and supported by modeling [11].

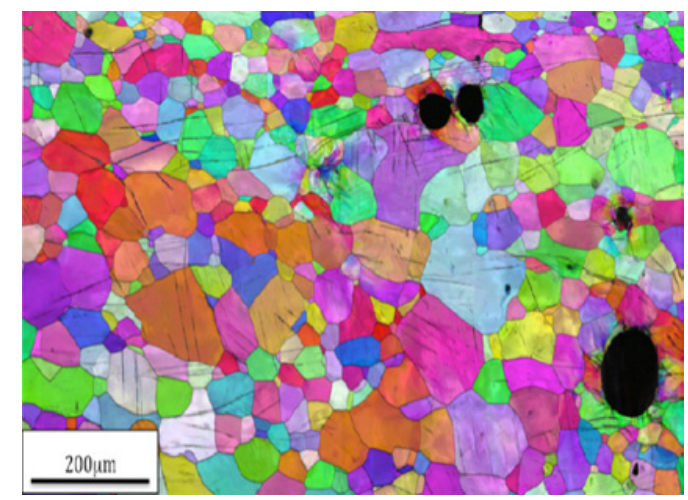

Fig. 9. Incipient damage in specimen $\boldsymbol{I I I}$ showing damage evolution below the main spall scab opening. The orientation triangle is the same as given in figure 5 .

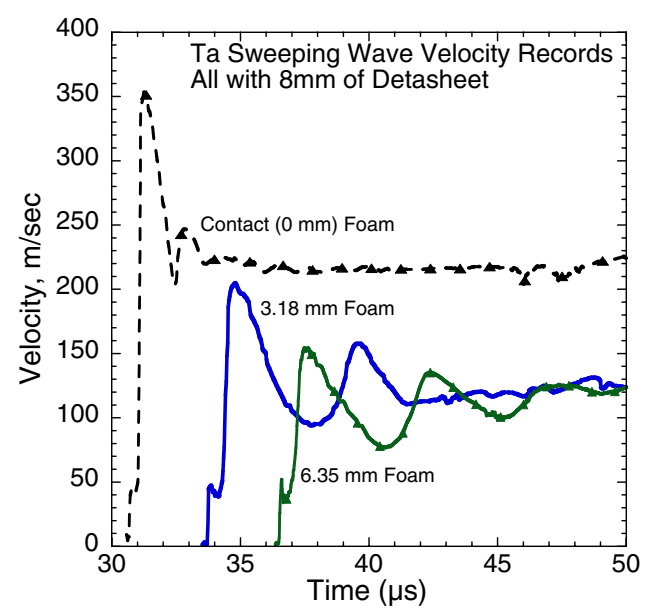

Fig. 10. PDV wave profiles (particle velocity versus time plot) for the three sweeping-wave-spallation experiments.

Removing the interlayer of foam in specimen $\boldsymbol{I I I}$ was seen to result in a single opened scab layer as well as a gradient of additional voids, void coalescence, shear localization, and cracking damage as a function of position from initiation of the sweeping wave. Figure 9 reveals a high density of fine deformation twins. Also observed, but not shown here, were large misorientations present below the opened scab fracture region after the sweeping wave was well established; i.e., approximately $2 / 3$ down the length of the sample. The high volume fraction of grains in which twins were activated is similar to previous observations of the influence of shock obliquity on the imposed local shear stresses in the Ta as previously documented [11].

\subsection{Velocimetry}

The PDV data for the three Ta sweeping-detonation-wave spallation experiments is presented in figure 10. The wave profiles for the three experiments are seen to vary as a function of the foam thickness for a fixed $8 \mathrm{~mm}$ of Detasheet of explosive. The effect of the removal of the foam in specimen $\boldsymbol{I I I}$ compared to specimens $\boldsymbol{I}$ and $\boldsymbol{I I}$ is evident in the increase of the jump-off velocity. As a result, the velocity achieved by each plate should be nominally 
Table 1. Sweeping-Wave Spall Shot Data; (*Specimen III spalled; the Romanchenko correction was only applied to this strength value).

\begin{tabular}{|l|c|c|c|c|c|}
\hline Sample & $\begin{array}{c}\text { Foam } \\
(\mathrm{mm})\end{array}$ & $\begin{array}{c}\text { Incident } \\
\text { Shock, } \\
\text { GPa }\end{array}$ & $\begin{array}{c}\text { Tensile } \\
\text { Spall } \\
\text { Stress, GPa* }\end{array}$ & $\begin{array}{c}\text { Strain } \\
\text { Rate, } \mathrm{s}^{-1}\end{array}$ & $\begin{array}{c}\text { Ringing } \\
(\mathrm{mm})\end{array}$ \\
\hline I & 6.35 & 4.5 & 2.6 & 4100 & 9.6 \\
\hline II & 3.18 & 6.0 & 3.8 & 6100 & 7.7 \\
\hline III & 0 & 10.6 & 5.4 & 24,000 & 2.5 \\
\hline
\end{tabular}

the same because the foam thickness variation is small. The in-contact experiment, specimen $\boldsymbol{I I I}$, shows a much higher "ultimate"velocity. This is because the plate spalled in this experiment. It should be noted that the velocity of the Hugoniot Elastic Limit (HEL) did not vary appreciably between the three experiments, but the character of the pull back due to yielding appears to be different. Data on the induced transverse velocities developed in Specimen I experiment has been discussed previously [14]. Similar data exists for specimens $\boldsymbol{I I}$ and $\boldsymbol{I I I}$ and is under analysis.

After the spall event, the velocimeter is measuring the velocity of the spall scab on the front surface rather than the entire sample. Conversely, in Specimens $\boldsymbol{I}$ and $\boldsymbol{I I}$ the plate remained intact and the velocimeter measured the lower ultimate velocity. As a beginning to the analysis of the data, the compressive stress of the incident shock, the tensile stress (pressure) achieved in the first unloading pulse, the strain rate of the unloading pulse, and the thickness implied by the duration of the period of the first ring, may be extracted from one of the probes positioned normal to the original surface. The formulas used are: $P=\rho_{0} u_{s} u_{p}$ for the incident pressure, $\sigma^{*}=\frac{1}{2} \rho_{0} c_{b} \Delta u_{f s}$ for the spall strength, $\dot{\varepsilon}=-u_{\mathrm{fs}}^{\bullet} /\left(2 c_{b}\right)$ for the strain rate just before spall occurs, and $h=\frac{\tau}{2} c_{l}$ for the implied thickness of the sample volume undergoing ringing. The Romanchenko correction [15] to the spall strength can be written as : $\Delta \sigma^{*}=\frac{h}{2}\left(\frac{d p}{d t}\right)\left(\frac{1}{c_{b}}-\frac{1}{c_{l}}\right)$ and the stress rate estimated from: $\frac{d p}{d t}=\rho_{0} c_{l} \dot{\varepsilon}$; The equation of state used is $u_{s}=3.402+1.22 u_{p}$ with $u_{p}=u_{f s} 2, \rho_{0}=16.65 \mathrm{~g} / \mathrm{cm}^{3}$, and $c_{b}=3.498 \mathrm{~mm} \mu \mathrm{s}$. The results are presented in Table 1

Analysis of the recovered sample indicates that Specimen III drastically spalled, and the implied spall scab thickness of $2.5 \mathrm{~mm}$ is consistent with the post-shot observations. Analysis of the recovered sample in Specimen $\boldsymbol{I}$ showed very little microscopic damage and was nominally intact. The implied thickness of the material undergoing ringing is calculated to be $9.6 \mathrm{~mm}$ is just slightly less than the original sample thickness of $10.2 \mathrm{~mm}$, and is also consistent with the post-shot and metallographic observations. Analysis of the recovered sample in Specimen II revealed some damage, but is also nominally intact. However, the implied thickness of the material undergoing ringing is calculated to be $7.7 \mathrm{~mm}$, which is significantly less than the original sample thickness. The first period of the ringing of the velocity in Specimen $\boldsymbol{I I}$ appears to have been interrupted early, and this interruption is responsible for the calculated intermediate thickness value. This disruption occurs before edge rarefactions should arrive, and is therefore likely associated with waves originating from damaged regions within the material, possibly from locations offset from the probe location. This analysis is consistent with the optical metallographic observation of a partially-formed spall plane and void coalescence in specimen $\boldsymbol{I I}$.

Finally, the experiment that showed pronounced spall, specimen $\boldsymbol{I I I}$, developed a tensile stress (spall strength of $\sim 5.4 \mathrm{GPa}$ for a $10.6 \mathrm{GPa}$ applied peak shock stress) below that quantified in previous studies of spallation in tantalum at equivalent peak shock stresses [16-20]. Previous 1D loading experiments have documented spall strengths of $5.2 \mathrm{GPa}$ for a $6 \mathrm{GPa}$ shock amplitude, a $7.3 \mathrm{GPa}$ spall strength for a peak shock stress of $9.5 \mathrm{GPa}[19,20]$, a spall strength of $6.2 \mathrm{GPa}$ after shock compression to 19 GPa. [21], and a spall strength of $8.1 \mathrm{GPa}$ Under quasiisentropic loading to $60 \mathrm{GPa}$ [18]. The lower apparent spall strength observed during sweeping-wave loading is postulated to reflect a change in the damage evolution in Ta from essentially pure void nucleation and growth to a higher contribution of shear-damage processes consistent with the increased imposed shear stresses commencesurate with the increased shear stresses, shock hardening, and increased twin formation propensity as a function of shock obliquity [11]. A higher propensity for twin formation with increasing obliquity coupled with the previous correlation of twins with void nucleation, may provide a plausible mechanism towards lowering spall strength. Further study is required to address the physical processes responsible for this decrease in spall strength.

\section{Summary}

The spallation response of Ta is demonstrated to be critically dependent on the amplitude of the sweeping-shockwave loading. Sweeping-wave loading is observed to: a) yield a lower spall strength than previously documented for 1-D supported-shock-wave loading, b) exhibit increased shock hardening as a function of increasing obliquity, and c) lead to increased incidence of deformation twin formation with shock obliquity. Simplistic models of spallation, such as $\mathrm{P}_{\text {min }}$ based on 1-D square-top shock data lack the physics to capture the influence of kinetics on damage evolution such as that operative during sweeping detonation loading. Quantification of the effects of shock obliquity on defect generation and damage evolution in shock loaded materials is critical to the development of physically-based models of the shock response of condensed matter.

\section{Acknowledgements}

Los Alamos National Laboratory is operated by Los Alamos National Security, LLC, for the National Nuclear Security Administration of the U.S. Department of Energy under contract DE-AC52-06NA25396. This work was partially sponsored by the Joint DoD/DOE Munitions Technology Development Program. 


\section{References}

1. G. T. Gray III, N. K. Bourne, B. L. Henrie: J. Appl. Phys. 101, 093507 (2007)

2. G. T. Gray III, L. M. Hull, J. R. Faulkner, M. E. Briggs, E. K. Cerreta, F. L. Addessio, N. K. Bourne, Shock Compression of Condensed Matter-2009 (American Institute of Physics Press, 2009)

3. G. T. Gray III, V. Livescu, E. K. Cerreta: Matls. Sci. Forum 654-656, 2297 (2010)

4. J. N. Johnson: J. Appl. Phys. 52, 2812 (1981)

5. G. T. Gray III, N. K. Bourne, B. L. Henrie, J. C. F. Millett: J. Phys. IV France 110, 773 (2003)

6. B. M. Butcher, L. M. Barker, D. E. Munson, C. D. Lundergan: AIAA Journal 2, 977 (1964)

7. J. S. Rinehart: J. Appl. Phys. 23, 1229 (1952)

8. L. V. Altshuler, S. A. Novikov, I. I. Divnov: Sov. Phys. Dokl. 11, 79 (1966)

9. W. E. Drummond: J. Appl. Phys. 29, 167 (1958)

10. V. A. Ogorodnikov, E. S. Tyunkin, A. A. Khokhlov, V. A. Grigorev, V. V. Mishukov: Strength Mater. 21, 1207 (1989)

11. G. T. Gray III, V. Livescu, E. K. Cerreta, T. A. Mason, P. J. Maudlin, J. A. Bingert, DYMAT 2009: 9th International Conference on the Mechanical and Physical Behaviour of Materials Under Dynamic Loading (EDP Sciences, 2009)

12. B. L. Adams, S. I. Wright, K. Kunze: Metallurgical Transactions A 24A, 819 (1993)

13. R. W. K. Honeycombe. The Plastic Deformation of Metals. Victoria, Australia: Edward Arnold Ltd., 1984
14. L. M. Hull, M. E. Briggs, J. R. Faulkner, Shock Compression of Condensed Matter-2011 (in press., 2011)

15. V. I. Romanchenko, G. V. Stepanov: Strength Mater. 9, 1110 (1977)

16. G. T. Gray III, In: High Pressure Science and Technology 1993, edited by Schmidt SC, Shaner JW, Samara GA, Ross M, American Institute of Physics, New York (1994), p. 1103.

17. J. N. Johnson, R. S. Hixson, D. L. Tonks, A. K. Zurek, In: Shock Compression of Condensed Matter 1995, edited by Schmidt SC, Tao WC, American Institute of Physics, Woodbury, New York (1996), p. 523.

18. L. C. Chhabildas, J. R. Asay, In: Shock-Wave and High-Strain-Rate Phenomena in Materials, edited by Meyers MA, Murr LE, Staudhammer KP, MarcelDekker, New York (1992), p. 947.

19. G. T. Gray III, A. D. Rollett, In: High Strain Rate Behavior of Refractory Metals and Alloys, edited by Asfahani R, Chen E, Crowson A, The Minerals, Metals and Materials Society, Warrendale, Pennsylvania (1992), p. 303.

20. A. K. Zurek, W. R. Thissell, J. N. Johnson, D. L. Tonks, R. Hixson: J. Mater. Process. Technol. 60, 261 (1996)

21. L. C. Chhabildas, L. M. Barker, J. R. Asay, T. G. Trucano, In: Shock Compression of Condensed Matter - 1989, edited by Schmidt SC, Johnson JN, Davidson LW, Elsevier, Amsterdam (1990), p. 429. 